\title{
Filigrane
}

Écoutes psychanalytiques

\section{Discussion du texte Bisexualité et différences des sexes de Catherine Chabert}

\section{Louise Mercier}

Volume 25, numéro 1, printemps 2016

Actes de la journée clinique de la SPQ (Catherine Chabert)

URI : https://id.erudit.org/iderudit/1037378ar

DOI : https://doi.org/10.7202/1037378ar

Aller au sommaire du numéro

Éditeur(s)

Revue Santé mentale au Québec

ISSN

1192-1412 (imprimé)

1911-4656 (numérique)

Découvrir la revue

Citer ce document

Mercier, L. (2016). Discussion du texte Bisexualité et différences des sexes de Catherine Chabert. Filigrane, 25(1), 119-127.

https://doi.org/10.7202/1037378ar d'utilisation que vous pouvez consulter en ligne. 


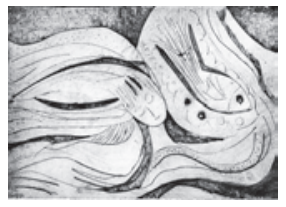

\section{Discussion du texte Bisexualité et différences des sexes de Catherine Chabert ${ }^{1}$}

\section{Louise Mercier}

L

ire un texte de Catherine Chabert, c'est se retrouver toujours au cour d'une - pensée riche et dense où les angles et les lignes de réflexion sont multiples. Un véritable tissage sans fin... Vous comprendrez que pour des fins de discussion, je tirerai quelques fils seulement, espérant dans l'après-coup qu'ils vous ouvrent sur le plaisir d'en tirer d'autres à votre tour.

J'aimerais souligner avant tout que c'est à travers son livre Féminin mélancolique que j'ai rencontré Catherine Chabert. Les nombreuses réflexions, questions et élaborations qu'elle amène dans ses différents écrits sur le féminin ont d'emblée été inspirantes pour moi. Cette façon singulière qu'elle a de nous transporter au cœur de l'inconscient avec son épaisseur à n'en plus finir, ses dérives, ses régressions trompe-l'œil et surtout mortifères, vient toujours nous rappeler et nous confronter à la violence du pulsionnel dont on ne peut faire l'économie en analyse. En même temps, son écriture nous relance constamment, nous remet au travail, crée du désordre et ainsi, se veut très féminine aussi. Elle ne ferme pas, elle n'enferme pas, elle reste ouverte, au travail. Il y avait de ce féminin-là chez Freud également.

Par ailleurs, la perlaboration oedipienne où s'élabore la différence des sexes et des générations a toujours occupé une place importante dans son travail. Là où la toute-puissance infantile et la position sado-masochiste occupent le premier plan du tableau clinique, là où le bourreau et la victime peuvent se donner longtemps la réplique avec les dérives mortifères dont elle sait si bien rendre compte, elle revient constamment aux résistances et aux embûches de la traversée oedipienne; là où, il faut se l'avouer, la partie est loin d'être gagnée. Pour l'analysant, sortir d'une position plus mégalomane ou accepter de ne plus être le bourreau ou la victime pour occuper une position de sujet, être responsable de sa vie avec tous les aléas qui peuvent 
se présenter, c'est être confronté à la profonde solitude porteuse à la fois de détresse et d'abandon, d'exclusion et de trahison et surtout de toutes les charges pulsionnelles qui lui sont inhérentes. C. Chabert en parle longuement et de multiples façons dans ses écrits. L'analyste, de son côté, n'est pas épargné pour autant. Accepter la passivité, accepter de rester au cœur de l'analyse, accepter de régresser à son tour pour occuper tous les rôles que l'analysant lui fait jouer, subir ses actings dans la régression partagée et par l'identification projective, tout cela l'amène bien souvent à son tour dans une position d'allure mélancolique. C'est là que l'espoir resté au fond de lui — ou au fond de la jarre nous dirait Pandora — peut venir ranimer ces moments où la détresse infantile n'est pas très loin, afin de lui permettre de la remettre à l'analysant.

De plus, tel que C. Chabert y revient dans ses écrits, mettre l'oedipe au centre de notre travail d'écoute n'est pas l'équivalent de faire faux bond à Winnicott ou à Ferenczi qui ont défriché à leur façon ce que Freud avait mis en friche et en élaboration suite aux limites soulevées par son travail sur l'hystérie. Au contraire, on sent toujours chez elle un profond respect pour eux. C'est plutôt son inquiétude qui est soulevée ici. En lien avec la tangente que peut prendre dans les différentes écoles analytiques la quasi exclusivité accordée à l'élaboration du maternel et/ou de l'objet primaire, elle souligne comment le maternel est soumis à son tour à la régression et à la déformation dans le suivi analytique. Se référant à Pontalis, elle nous rappelle alors qu'il proposait de se tourner vers la mère, certes, mais sans se détourner du père pour autant. De façon similaire, Green affirme, dans La mère morte: «L'oedipe doit être maintenu comme matrice symbolique essentielle à laquelle il est important de toujours se référer même dans les cas où la régression est dite pré-génitale ou pré-oedipienne... Si poussée que soit l'analyse du désinvestissement de l'objet primaire, le destin de la psyché humaine est toujours d'avoir deux objets et jamais un seul, si loin que l'on recule pour essayer de cerner la structure psychique dite la plus primitive.» (1983, p. 227).

\section{La bisexualité}

Nous sommes ici convoqués au cœur du travail de C. Chabert où l'analyste et la théoricienne en elle se donnent la main. La thématique du colloque consistait en «L'amour de la différence», mais c'est la «haine de la différence» qu'elle évoque ici en proposant de discuter de la bisexualité et de la différence des sexes. De plus, intuition théorique féconde s'il en est, même si la bisexualité psychique peut brouiller les cartes parce qu'elle serait présente 
d'emblée dès la naissance, pour les deux sexes, y revenir sous-entend rester à la fois au coeur du sexuel infantile et des identifications maternelles et paternelles. En d'autres termes: la mère oui, mais le père également. La scène primitive n'est donc pas très loin à l'arrière-plan.

Comme C. Chabert le souligne à la suite de Beetscheen, l'infantile est sexuel et toujours agissant. Il doit affronter la différence dans sa dimension d'altérité et dans la différence des sexes et des générations. La bisexualité psychique (masculin et féminin) inhérente aux deux sexes peut en ce sens se mettre au service de l'élaboration d'identifications oedipiennes inscrites dans la différence des sexes - la "chose sexuelle», nous dirait Nathalie Zaltzman -, mais elle peut aussi venir défensivement s'arrimer au pendant narcissique de la psychosexualité dans une quête effrénée de fusion. Être tout, être parfait, sans manque, sans attente... Comme ce fut le cas de l'une de mes analysantes aux prises avec «une envie de pénis» impressionnante (forte charge hallucinatoire) qui était certaine qu'un garçon comblerait à la fois son père et sa mère (être tout pour papa, être tout pour maman), tentant de nier du même coup la scène primitive. Il nous a fallu traverser bien des tempêtes transféro-contre-transférentielles, beaucoup d'impuissance aussi, pour qu'elle se découvre en fin de parcours «juste une femme», mais paradoxalement «toute une femme», sortie de l'emprise d'identifications à des figures parentales fortement sexualisées, d'allure perverse, culpabilisante et mortifère, faisant écho aux élaborations de C. Chabert sur le Surmoi féminin, régressé, faible et cruel.

En fait, c'est de cela aussi dont il est question dans les vignettes cliniques que C. Chabert présente. Elle nous rappelle, encore une fois à l'instar de Beetscheen, comment le surinvestissement de la bisexualité peut offrir un relais à la violence de la différence des sexes, à l'horreur de l'exclusion, aux excitations et aux désirs trop débordants qu'elle peut susciter. Mais à quel prix? Comme pour les deux analysants que l'auteure évoque, qui surinvestissent leur contraire afin de rester au cœur du fusionnel/incestuel, mais au détriment de leurs aspirations amoureuses et familiales.

Suivons-les d'un peu plus près... C. Chabert nous fait bien sentir de prime abord comment leur différence de sexe vient jouer dans la façon qu'ils ont d'aborder leur démarche, de mettre en scène et d'élaborer dans le transfert leur bisexualité. Mais dans ce long travail où son impuissance, mise à rude épreuve, devient son phare d'interprétation, alors que la perlaboration ouvre sur des avancées et leur permet enfin de contacter leur solitude et leur détresse d'adolescence, leurs résistances s'affirment à nouveau. Retour en 
force de leur bisexualité psychique pour rester au cœur de l'attachement aux figures parentales, incapables de choisir. Dans la lecture, nous voilà confrontés à notre tour à des relents d'impuissance et à l'ampleur du travail qui reste à faire. Comme si, incapables dans le transfert d'élaborer leur haine inhérente à la traversée oedipienne et soulevée par les absences de l'analyste, c'est contre eux finalement que cette agressivité (haine) se retourne dans une auto-critique d'allure mélancolique qui vient bafouer leurs projets de vie dont l'analyse, entre autres, fait partie.

On est loin, dans ce cas, d'une appropriation subjective de l'attente liée à «l'entre-eux-deux», attente qui permettrait le mouvement de l'un à l'autre, le détour et l'ouverture rêvante, nous dirait C. Chabert. On pourrait penser d'ailleurs que chez ces analysants, la fixation à la bisexualité, en plus de constituer un havre contre la scène primitive, témoignerait également d'un besoin d'asile pour un moi en quête de protection; là où le risque de détruire ou de perdre l'objet est davantage narcissique et plus près d'une pulsionnalité proche de l'auto-conservation. C'est ici que la référence à Winnicott (1947) s'avère pertinente, me semble-t-il, lorsqu'il aborde l'importance de survivre à la haine de l'analysant, dans ces zones de régression où l'objet total n'est pas pleinement constitué, chez les névrotiques également. Martin Gauthier (2013), dans son article «Origine meurtrière du Surmoi», souligne notamment l'importance de la survie de l'objet pour permettre, du côté de l'analysant, l'appropriation subjective du caractère impitoyable de la charge pulsionnelle de ses matières meurtrières. En bref, il s'agirait de permettre l'accomplissement du meurtre afin qu'il trouve toute sa portée symbolique. Et si l'on ajoute que dans ce long travail de digestion, de manière défensive, les identifications primaires et oedipiennes peuvent longtemps se renvoyer la balle, c'est dire combien le travail de perlaboration peut être long, exigeant, et porteur de questionnements.

\section{Le féminin pur}

Ici, C. Chabert, la théoricienne, refait surface. Elle questionne entre autres les écueils possibles de la bisexualité psychique en se demandant de quel féminin au juste parle-t-on en examinant le féminin des deux sexes: identifications primaires/socle de la construction identitaire ou identifications oedipiennes? Elle revient alors sur le «féminin pur» de Winnicott (1966) dans la bisexualité. Elle nous rappelle que ce dernier en parle comme d'un féminin pur à l'origine du Soi, qui permet l'expérience d'Être: «Le bébé devient le sein, l'objet est dans le sein, il n'y a là aucune motion pulsionnelle 
car l'objet n'est pas constitué.» Le pulsionnel viendrait après, avec le «Faire» nous dit-il, qui lui est masculin. C. Chabert questionne ce féminin pur non contaminé, dépulsionnalisé (donc pourquoi féminin?). Alors qu'elle met en doute l'existence du clivage féminin pur proposé par Winnicott (« $\mathrm{Ne}$ retrouverait-on pas dans cette bataille [...] l'actualisation d'une scène primitive condensant toujours identification et choix d'objet?»), elle souligne qu'il ne s'accorderait pas avec la bisexualité de Freud qui en parle comme «bien plus de l'un que de l'autre», donc inscrit dans la pulsionnalité et la différence des sexes.

Pour ma part, à la lecture de cet article de Winnicott, je me suis d'abord demandé si «cette envie du pénis» de son analysant ne nous parlait pas tant d'un féminin pur que d'un désir chez lui de pouvoir jouir de son pénis, de sa masculinité, donc inscrit dans la bisexualité psychique. En poursuivant ma lecture, j'ai alors eu l'impression de comprendre, autant que faire se peut, que pour Winnicott, ce féminin clivé ( «je suis une fille») serait pathologique et lié à l'impossibilité pour l'analysant de vivre l'expérience du «féminin pur» (être le sein) qui elle permettrait l'expérience d'Être et l'intégration éventuelle de la bisexualité. Comme si ce féminin là était antérieur au féminin/masculin de la bisexualité. Il serait tentant ici de faire un parallèle entre l'émergence du Soi de Winnicott, lié à l'expérience d'Être, et le noyau du narcissisme primaire freudien. Cléopâtre Athanassiou-Popesco (2004) en discute, d'ailleurs, dans le livre Winnicott insolite. Ce noyau qui, pour se constituer, demande une satisfaction réelle avec l'objet, donc un objet lui-même différencié et pulsionnalisé. Winnicott, en évoquant le féminin pur, se demande s'il ne s'agit pas de l'identification primaire. Ainsi, loin d'être a-pulsionnel, ce féminin s'approcherait de l'expérience du féminin antérieure aux représentations, au plus près du pulsionnel, de l'affect et de l'éprouvé, point de sédimentation de la bisexualité et des identifications, comme C. Chabert le rappelle dans Féminin mélancolique. Cette expérience du féminin, ne pourrait-on pas en parler comme d'un premier mouvement (en référence au pictogramme de Piera Aulagnier) qui viendrait marquer en positif ou en négatif le féminin/masculin de la bisexualité; une expérience d'emblée présente (par les parents ou par le père dans la mère) mais surtout, une expérience à élaborer, à construire? Du reste, la question demeure: pourquoi «pur»? D’autant plus que Winnicott (1966) évoquera également des éléments masculins purs. On en vient à se demander s'il s'agit pour lui d'une pureté a-pulsionnelle, ou s'il ne s'agit pas plutôt d'une pure expérience du féminin et du masculin. 
Sans reprendre ici tous ses développements, soulignons que C. Chabert en vient alors à interroger le féminin de la bisexualité chez l'homme et chez la femme en analyse. Elle s'inspire cette fois de Freud et de Pontalis, pour l'inscrire à son tour dans la différence des sexes et la conflictualité sousjacente (amour/haine) qui serait évitée par l'entremise du féminin pur de Winnicott. Tout pour éviter le conflit, tout pour ne pas choisir. C'est ce que l'on observerait entre autres avec ses deux analysants, encore là de façon différente; elle, dotant fantasmatiquement sa mère d'un féminin pur, mère idéale, asexuelle, nullement rivale, nullement abandonnique ou rejetante (toute l'élaboration de la naissance du petit frère est évitée ici); lui, en clivant ses choix de femmes, femme tranquille, douce et apaisante d'un côté (contre-investissement de la mère trop excitante et pulsionnelle qu'il aurait eue) et femme légère de l'autre. Dans les deux cas, l'idéalisation de la mère est maintenue, féminin pur, culte de la Vierge à l'abri de la haine et du mépris.

Dans les deux cas également, mais de façon différente, la bisexualité psychique soutenue par une «identification féminine clivée à la Winnicott» viendrait contre-investir la scène primitive, mais au prix d'une bien grande culpabilité. Comme l'avançait Beetscheen, cela reste violent malgré tout. Bisexualité culpabilisante pour elle, aux prises avec une identification au père fortement incestuelle; bisexualité castrante pour lui, où assumer sa virilité revient à s'identifier à un père bourreau (en écho, l'on peut penser au petit garçon bourreau qu'il était vis-à-vis son jeune frère). De là, les nouvelles interrogations de C. Chabert sur le Surmoi au féminin, autre écueil de la bisexualité. Loin du féminin pur de Winnicott, elle se demande si cette désexualisation maternelle n'incarnerait pas plutôt «un moi idéal défendu farouchement par un Surmoi sévère qui empêcherait l'accès au plaisir et à la jouissance sexuelle».

\section{Surmoi au féminin}

En lien au déclin et à la destruction de l'oedipe issus de la crainte de la castration, C. Chabert nous rappelle que pour Freud, il y aurait chez l'homme un «bien plus de Surmoi», à entendre comme un Surmoi plus fort, bienveillant et protecteur. Je dois avouer que j'ai toujours été saisie par ce développement chez Freud, cette destruction de l'oedipe chez le garçon due à la castration. Comme s'il disparaissait quasi magiquement alors que lui aussi est soumis à la rivalité, la haine et la déception. Interprétée ainsi, on a l'impression que cette destruction prend des allures défensives qui viendraient éclipser la traversée oedipienne. Comme si Freud, de façon 
différente de Winnicott, malgré son «complexe paternel» et ses théorisations sur l'oedipe, avait clivé à son tour le masculin et le féminin. Tous deux, à leur façon, faisant l'économie de la scène primitive en maintenant inconsciemment intacte pour l'un l'image de la mère adorée et pour l'autre, une réparation éternelle de sa «Mère morte». Exit le père.

Revenant à Freud, C. Chabert souligne alors que pour lui, la femme, poussée vers le père, resterait longtemps dans l'oedipe et son Surmoi en pâtirait. Faiblesse du Surmoi au féminin, différent certes, mais proche dans sa sévérité du Surmoi de Melanie Klein, nous propose-t-elle. Elle reprend ce Surmoi au féminin de Freud pour en parler plutôt comme d'un Surmoi susceptible de se rencontrer chez l'homme, également en lien à la bisexualité. C'est en référence à ce Surmoi qu'elle comprend les régressions et les dérives mortifères qui, en dépit ou à cause des progrès en analyse, ressurgissent de façon régulière chez ses analysants. En plus de se nourrir du masochisme du Moi, il leur a permis la transgression (par exemple, ne pas payer les séances manquées).

\section{Quelques réflexions supplémentaires}

Je ne sais pas où en sont rendus aujourd'hui ces deux analysants mais j'ai l'impression que malgré leur désir récurrent de mettre un terme au suivi, leurs démarches respectives se poursuivent toujours. On les sent tous deux au cœur du travail analytique et souvent, j'ai eu l'intuition que les deux identifications, à la fois maternelles et paternelles, étaient condensées et travaillées en même temps dans le transfert. Lorsque C. Chabert explique que son analysante voulait la séduire et qu'en lien aux séances manquées qu'elle devait payer, elle voulait avoir des passe-droits pour retrouver cette place privilégiée auprès de sa mère (d'avant son frère), de mon côté, en lisant l'anecdote, je me demande si dans le transfert, elle n'est pas également le père que son analysante voulait séduire par ses réalisations intellectuelles. Je veux souligner ici combien dans de telles configurations, le travail de condensation peut être porteur de confusion; l'analyste se demande alors qui il représente dans le transfert et doit accepter de porter simultanément différents chapeaux.

Le texte de C. Chabert démontre bien, aussi, comment la perception de la réalité vient doubler le travail sur la scène primitive, scène sado-masochiste en soi. Je pense ici aux scènes violentes qui semblent avoir marqué la vie de son analysant, rendant encore plus difficile le travail sur les résistances (son cheminement m'apparaît d'ailleurs plus compliqué et tortueux 
que celui de l'analysante évoquée). En ce sens, la bisexualité serait travaillée de façon différente par la différence des sexes, mais les relations parentales perçues teinteraient également le processus analytique.

Enfin, je me questionne sur la bisexualité post-oedipienne (toujours en mouvement, jamais figée) dans sa composante féminine au terme d'une analyse. Ici, je pense à nouveau à mon analysante. Elle qui avait fait des études doctorales pour me plaire comme un garçon, réalisant douloureusement au fil du suivi qu'elle n'aurait ni pénis, ni place privilégiée, me dira quelques semaines avant son départ: «Vous savez, moi qui voulais vous épater comme un garçon avec mon doctorat, je réalise finalement que tout ça vient servir mon identité féminine.» Et puis, très touchée, lors d'une des dernières séances: «Avant je n'étais qu'un trou dans lequel il venait jouir. Maintenant je sais que je peux jouir et que je peux le faire jouir.»Une bisexualité empreinte d'un bien plus de féminin et d'un bien plus de Surmoi nous dirait Freud. Un Surmoi au masculin pourrions-nous dire? Celui qui s'est construit dans la relation transféro-contre-transférentielle, au cour de la position dépressive, entraînant progressivement avec lui les figures parentales idéalisées, trop sexualisées, et témoignant de nouvelles identifications. Surmoi plus protecteur (qui n'est sûrement pas à l'abri lui non plus de la régression), identité féminine affermie, là où la haine et l'envie (du pénis) a fait place au désir et à l'amour de la différence.

\section{Conclusion}

Comme notre invitée nous le rappelle à l'occasion dans ses écrits, Freud nous disait: "Jamais nous ne sommes autant privés de protection contre la souffrance que lorsque nous aimons, jamais nous ne sommes davantage dans le malheur ou la détresse que lorsque nous avons perdu l'objet aimé et son amour». C'est dire l'entreprise de notre travail analytique.

Dans ses écrits comme dans ses conférences, Catherine Chabert nous donne l'occasion de pousser plus loin nos réflexions et alimente en nous l'espoir qui nous porte comme cliniciens. En dépit de la passion et du plaisir qui nous animent, nous en avons toutes et tous un grand besoin.

Louise Mercier louisemercier9@gmail.com 


\section{Note}

1. Présenté à l'occasion de la journée clinique d'octobre 2015 organisée par la Société psychanalytique de Québec, à la suite de la conférence de Catherine Chabert.

\section{Références}

Athanassiou-Popesco, C. (2004). Le noyau narcissique primaire. Ses rapports avec le vrai Self winnicottien. Dans J. Boushira et M.-C. Durieux (dir.), Winnicott Insolite (p.109-133). Paris: Presses universitaires de France.

Chabert, C. (2003). Féminin mélancolique. Paris: Presses universitaires de France.

Chabert, C. (2011). L'amour de la différence. Paris: Presses universitaires de France.

Freud, S. (1930). Le malaise dans la culture. Dans Euvres complètes Tome XVIII 1926-1930 (p. 245-334). Paris: Presses universitaires de France, 2002.

Gauthier, M. (2013). Origine meurtrière du Surmoi. Revue Française de psychanalyse, 77 (5) 1488-1494.

Green, A. (1983) La mère morte. Dans Narcissisme de vie, Narcissisme de mort (p. 222-253). Paris: Les Éditions de Minuit.

Pontalis, J.-B. (1977). Entre le rêve et la douleur. Paris: Gallimard.

Winnicott, D.W. (1966). Clivage des éléments masculins et féminins chez l'homme et chez la femme. Nouvelle Revue de psychanalyse, (7), 301-315.

Winnicott, D.W. (1947). La haine dans le contre-transfert. Dans De la pédiatrie à la psychanalyse (2 éd., p. 48-58). Paris: Payot, 1969. 